\title{
Acento léxico y lectura: un estudio con niños
}

\author{
Nicolás Gutiérrez Palma ${ }^{1}$, Alfonso Palma Reyes ${ }^{2}$ \\ ${ }^{1}$ Departamento de Psicología, Universidad de Jaén \\ ${ }^{2}$ Dpto. de Psicología Experimental y Fisiología del Comportamiento, \\ Universidad de Granada
}

\section{España}




\section{Resumen}

Introducción: El acento en español se asocia con una marca ortográfica que lo indica, pero también hay otras pistas que lo señalan. La mayoría de las palabras se acentúan de la misma forma (como llanas) y las sílabas pesadas atraen el acento. En este trabajo se estudian estas pistas y, a través de ellas, la función del acento como uno de los códigos fonológicos que intervienen en el proceso de la lectura.

Método: El estudio se realizó con niños con poca experiencia lectora, mucho más propensos al uso de la ruta fonológica. Se utilizó una tarea de naming de palabras, y se manipuló el acento, así como la regularidad-irregularidad de la estructura silábica respecto al mismo.

Resultados: No hubo diferencias entre las palabras según fuese su acento, llano o agudo. Sin embargo, sí que las hubo según fuesen regulares o irregulares. Las primeras se leyeron con menos errores.

Discusión: Este resultado se interpretó como una prueba de la relación funcional del acento con la estructura silábica, así como de su papel en el proceso de acceso al léxico que se realiza durante la lectura. Finalmente, se discute la conveniencia de incluir las variables prosódicas como parte de los programas para el entrenamiento de la conciencia fonológica.

Palabras Clave: acento léxico, acceso léxico, lectura 


\section{Introducción}

Leer es una actividad compleja, tanto por la cantidad como por la diversidad de las operaciones cognitivas que intervienen en ella. En términos muy generales, los tipos de procesos que se realizan son los siguientes. Inicialmente, los procesos de análisis visual se encargan de identificar las letras que forman las palabras. Les siguen los procesos de acceso al léxico, que buscan el significado y otras características de las palabras en el léxico mental. Posteriormente, los procesos sintácticos agrupan las palabras en sintagmas y a éstos entre sí, además de determinar el papel que cada sintagma juega dentro de la oración. Finalmente, los procesos semánticos cumplen la función de extraer el significado del texto y de integrar esa información con el resto de los conocimientos almacenados en la memoria a largo plazo. Dentro de este marco teórico, la investigación que aquí se presenta se centra en el acento como uno de los códigos fonológicos que los niños pueden utilizar a modo de pista para acceder al léxico de las palabras escritas.

\section{Acento y acceso al léxico}

Hasta la fecha se han acumulado pruebas suficientes que indican que el proceso de acceso al léxico está mediado fonológicamente (Frost, 1998). Algunos de los códigos fonológicos que se han propuesto se refieren a los fonemas y a los grupos de fonemas (p.e., Coltheart, 1978), así como a las sílabas (p.e., Carreiras, Álvarez y de Vega, 1993; Domínguez, de Vega y Cuetos, 1997; Álvarez, de Vega, y Carreiras, 1998; Álvarez, Carreiras y de Vega, 2000). Sin embargo, en español hay otros códigos posibles. El acento es en algunos casos la única pista de la que se dispone para distinguir entre palabras parecidas (p. e., mato y mató, saco y sacó, sábana y sabana, jugo y jugó, etc.), y de ahí el interés de su estudio. Por otra parte, se ha demostrado su papel en el acceso al léxico que se realiza en la modalidad auditiva (Cutler, Dahan y Donselaar, 1997; Soto, Sebastián-Gallés y Cutler, 2001). Si se parte de la posibilidad de que leer y escuchar una palabra conlleve el uso de una representación fonológica común (Borowsky, Owen y Fonos, 1999; Haist, Song, Wild, Faber, Popp, y Morris, 2001), hay razones para pensar que el acento forme parte de ella y que pueda influir en los procesos de reconocimiento también en la modalidad escrita.

En el supuesto de que la hipótesis anterior sea cierta, la siguiente cuestión que debe plantearse se refiere a las pistas que indican el acento en la lengua escrita. La más evidente es la tilde, cuya relación con el acento es perfecta. Le sigue el conocimiento implícito de los lec- 
tores acerca del acento más frecuente, que en español es el acento llano (Harris, 1995). Por último, la estructura silábica también indica el acento según la regla de que se acentúa la penúltima sílaba, a menos que la última sea pesada (Alonso-Cortés, 1998). En este trabajo se va a estudiar el uso que hacen los lectores noveles de esta información, sobretodo en relación a las dos últimas pistas.

\section{Pruebas del papel del acento en el proceso de acceso al léxico}

La investigación realizada sobre este tema aporta pruebas de que el acento se asigna según las pistas ya descritas en el párrafo anterior. En cuanto a la tilde, Domínguez y Cuetos $(1998,2002)$ manipularon el acento (inducido por la tilde) de dos palabras que se presentaban seguidas (esto es, según un procedimiento de priming). En una condición, de identidad, coincidió tanto su acento como su ortografía ( $R A S G O$ - rasgo). En otra condición, de acento incongruente, sólo coincidió la ortografía ( $R A S G O ́$-rasgo). Finalmente, en la condición control, o de acento congruente, únicamente coincidió el acento (PERSA-rasgo). Además, manipularon el tiempo que tardaba en aparecer la segunda palabra en relación al comienzo de la primera (esto es, el SOA), y la tarea que utilizaron fue la de decisión léxica. En el primer experimento (con un SOA de $32 \mathrm{~ms}$ ) encontraron que la condición de identidad fue más rápida que la de acento incongruente ( $R A S G O$-rasgo frente a $R A S G O$-rasgo), mientras que no hubo diferencias entre las condiciones de acento congruente e incongruente (PERSA-rasgo frente a $R A S G O ́$ - rasgo). Interpretaron este resultado argumentando que no hubo diferencias porque en ambas condiciones pudieron haberse sumado, en sentido contrario, efectos de la misma magnitud. En la condición de acento incongruente, la facilitación debida a la ortografía de la palabra pudo haber sido contrarrestada por la inhibición debida al acento. En la condición control pudo haber ocurrido lo contrario. Para comprobarlo, Domínguez y Cuetos compararon la condición incongruente con dos situaciones control (experimento 2). En una de ellas, las dos palabras compartieron su acento (PERSA-rasgo), mientras que en la otra no (DORMÍrasgo). La comparación entre la condición incongruente y el segundo control permitió aislar el componente ortográfico, mientras que la comparación entre los dos controles sirvió para aislar el componente prosódico. En el primer caso (RASGÓ-rasgo frente a DORMÍ-rasgo) los tiempos de reacción fueron menores en la condición incongruente. En el segundo caso (PERSA-rasgo frente a DORMÍ-rasgo) hubo una ventaja del primer control que sugiere que el acento influye en el proceso de acceso al léxico desde etapas muy tempranas. 
Además de la tilde, hay otros caracteres gráficos que se relacionan con el acento en otros idiomas. Kelly, Morris y Verrekia (1998) así lo indicaron para el caso del inglés. Por ejemplo, la presencia de más letras de las necesarias para representar el fonema final de una palabra cara a su pronunciación es una marca para el acento agudo en este idioma. Tal es el caso del vocablo discuss, en el que la última $s$ no es necesaria para la pronunciación del fonema $/ s /$ pero que la marca como aguda, frente a discus en que la ausencia de la letra $s$ la marca como llana. Por lo tanto, algunas letras en posición final podrían considerarse análogas en función a la tilde española, aunque su relación con el acento no sea perfecta. Kelly et al. estudiaron lo que sucedía cuando no se cumplía esta regla en las palabras que, pese a estar marcadas como agudas, eran llanas. Para ello compararon las palabras marcadas frente a las no marcadas a través de las tareas de decisión léxica y de denominación de palabras. El resultado fue que los tiempos de reacción fueron superiores en el caso de las marcadas, lo que fue interpretado por los autores como una prueba de que el acento interviene de alguna forma en el proceso de acceso al léxico que se realiza en inglés.

El conocimiento implícito de los lectores acerca de cuál es el acento más frecuente también ha demostrado ser una pista relevante, aunque la investigación realizada ha producido resultados contradictorios. En la mayoría de los trabajos realizados la manipulación principal ha consistido en comparar los vocablos según fuese la frecuencia de su acento (por ejemplo, las palabras llanas frente a las agudas o las esdrújulas en español). El resultado más habitual cuando se utiliza la tarea de denominación de palabras es que los tiempos de reacción sean menores en las palabras de acento más frecuente (Colombo, 1992; Sbisa, Zorzi y Tabossi, 1998), y que con la tarea de decisión léxica se cometan menos errores (Black y Byng, 1986; Colombo, 1992; Gutiérrez, Palma y Santiago, 1998). Estos resultados sugieren que los lectores asignan un acento por defecto que corresponde al más frecuente de su idioma. Sin embargo, también hay pruebas en contra de esta hipótesis. Cuando se comparan dos listas de palabras, según tengan todas el mismo acento o éste sea variable, se encuentra que no hay diferencias (Cutler y Clifton, 1984). Por lo tanto, conocer de antemano el acento de una palabra no supone ninguna ventaja. Este resultado se ha encontrado en inglés, y cuestiona que el acento cumpla ninguna función para el acceso al léxico en este idioma.

Además de la tilde y del acento más frecuente, la estructura silábica es otro factor a tener en cuenta dada su estrecha relación con el acento. De acuerdo con ello, hay pruebas que indican que esta última es una buena pista que se utiliza para asignar el acento de las palabras escritas. Miceli y Caramazza (1993) estudiaron el caso de un paciente diagnosticado de dis- 
lexia adquirida que tenía deteriorada su habilidad para acceder al léxico fonológico, si bien todavía conservaba la capacidad de utilizar reglas. Cuando la tarea consistió en leer palabras en voz alta, su paciente cometió más errores en las irregulares que lo eran por su acento, es decir, aquellas en las que éste no coincidía con el que debían tener por su estructura silábica (por ejemplo cáliz, que al ser CV-CVC debía acentuarse como aguda). Posteriormente, Cappa, Nespor, Ielasi y Miozzo (1997) encontraron el mismo resultado con un paciente afásico. Ambos estudios se hicieron en italiano, y con pacientes que habían sufrido una lesión cerebral. Cabe preguntarse, por lo tanto, por lo que sucedería en otros idiomas y con sujetos normales. En respuesta a esta pregunta, Gutiérrez (2003) obtuvo resultados semejantes en español. Al comparar palabras regulares (por ejemplo, casa, que por su estructura $\mathrm{CV}-\mathrm{CV}$ tiene que ser llana) e irregulares (por ejemplo cáliz), encontró que se cometían más errores en el segundo caso que en el primero y que, además, esas diferencias desaparecían cuando las palabras se escribían con tilde.

\section{Acento y lectura en niños}

Hasta donde nosotros conocemos, no hay estudios que hayan analizado esta problemática en la población infantil. Sin embargo, hay pruebas indirectas que justifican la investigación que se plantea en este trabajo. Por una parte, se sabe que los niños aprenden muy pronto las reglas para la asignación del acento en el habla, y que no se limitan sólo a memorizar los acentos de las palabras (Hochberg, 1987a, 1987b). Por otra parte, hay pruebas de que los niños españoles se sirven de la estructura silábica para leer. Consecuentemente, cometen más errores en las palabras de estructura silábica más compleja. Esto sucede en niños de entre 7 y 12 años de edad, con independencia de su competencia lectora (Defior, Justicia y Martos, 1996).

Dado que los niños conocen las reglas para la asignación del acento en el lenguaje oral y, además, su lectura se ve afectada por la estructura silábica, es factible que aprendan relativamente pronto la relación entre el acento y la estructura silábica en la lengua escrita. Esta posibilidad fue comprobada recientemente por Gutiérrez (en prensa) quien utilizó niños de segundo curso de primaria para comparar palabras regulares e irregulares (según su relación estructura-acento) a través de una tarea de decisión léxica. El resultado fue que las palabras regulares (p. e., calor) se reconocieron con menos errores que las irregulares (p. e., café). Las diferencias encontradas sólo fueron marginalmente significativas, aunque en un análisis de regresión posterior se obtuvo una correlación positiva (sólo para las palabras regulares) entre 
los errores cometidos en la tarea y en una prueba previa de lectura de no-palabras. Los errores en la lectura de no-palabras son un índice del conocimiento de las reglas fonológicas, por lo tanto es lógico que a más errores se cometan en ellas también se produzcan más errores en las palabras que siguen las reglas. Cabe esperar, sin embargo, que dicha relación no se encuentre en las palabras irregulares, que son las excepciones. Eso fue exactamente lo que sucedió.

\section{Modelos de acceso al léxico que incorporan el acento}

Hasta donde nosotros sabemos, los modelos de Black y Byng (1986), y de Rastle y Coltheart (2000) son los únicos que integran el acento en alguna de las fases del proceso de acceso al léxico. Ambos son modelos de doble ruta, pero difieren en sus propuestas acerca de cómo se asigna y utiliza el acento. En el modelo de Black y Byng (1986), el procedimiento consiste en contar las vocales de las palabras para identificar sus sílabas. Esa es la única información necesaria, dado que el acento se define por la relación (fuerte-débil) que se establece entre ellas. Posteriormente, se asigna el acento que sea más frecuente para ese número de sílabas (p. e., el acento llano ${ }^{1}$ ). Utilizando dicho acento como guía, la búsqueda léxica comienza por la sílaba acentuada y, sólo después, se tiene en cuenta el resto de la información fonológica y semántica. Si en un primer ciclo se activa la entrada léxica adecuada, el proceso se detiene, si no, se repite todo el ciclo con el siguiente acento en orden de frecuencia.

En el modelo de Rastle y Coltheart (2000), la asignación del acento parte de un análisis morfológico de la palabra. El procedimiento, según las regularidades de la lengua inglesa, sería el siguiente. En primer lugar, se buscan los prefijos. Cuando se detectan se recupera su pronunciación directamente del léxico, mientras que el resto de la palabra se transcribe conforme a las reglas de conversión grafema-fonema (CGF a partir de ahora). Una vez hecho esto se comprueba el resultado final, para evitar que los dos últimos elementos de la cadena de fonemas violen alguna regla fonotáctica. $\mathrm{Si}$ se quebrantase alguna regla, se acentuaría la última sílaba, si no, se aplicarían las reglas CGF a la palabra completa, y se asignaría el acento a la primera sílaba. Si no se encuentran prefijos se buscan sufijos. Dado que es posible que haya más de uno, este procedimiento se repite varias veces (sería recursivo) hasta que todos los sufijos sean identificados. Si se detectara uno, se buscaría su pronunciación en el léxico y, salvo algunos casos (como en los sufijos -een, -ique y -oo), el acento se asignaría a la primera sílaba. El resto de la palabra se transcribiría conforme a las reglas CGF. Si, por el contrario, no hubiese sufijos, se acentuaría la primera sílaba y se aplicarían las reglas CGF al resto de la palabra. 
Rastle y Coltheart encontraron que, sobre un corpus de 23266 entradas, el algoritmo asignaba el acento correctamente en el $89.7 \%$ de los casos, y en el 95\% de las palabras llanas. Por otra parte, en una tarea de lectura de no-palabras, el acento predicho coincidió con el observado en un buen número de casos (experimento 2). Finalmente, las palabras regulares (cuyo acento coincidía con el que hubiera asignado el algoritmo) se leyeron más rápido y con menos errores que las irregulares (cuyo acento no coincidía). Estas diferencias fueron más acusadas en el caso de las palabras de baja frecuencia (experimento 3 ).

Este algoritmo sustituye a la ruta no-léxica en el modelo DRC (Coltheart y Rastle, 1994; Rastle y Coltheart, 1998, 1999, 2000). La propuesta de un algoritmo específico para el caso del español va más allá de los objetivos de este trabajo, sin embargo sí puede decirse que cualquiera que se proponga debería considerar los factores que pueden influir en la asignación del acento en este idioma. Entre ellos, tal y como ya se ha expuesto, están que la mayoría de las palabras sean llanas y que las sílabas pesadas atraigan el acento. Ambos factores se combinan en la norma general de que el acento recae en la penúltima sílaba, a menos que la última sea pesada, en cuyo caso ésta recibe el acento (Alonso-Cortés, 1998). Por lo tanto, parecería que cualquier algoritmo que se propusiera debería trabajar desde el final de la palabra. Sin embargo, ello llevaría a errores en aquellos casos en los que el acento viniera indicado al principio de la palabra a través de la tilde. Para evitarlo, la dirección tendría que ser de izquierda a derecha, y sílaba a sílaba ${ }^{2}$. En este trabajo, se quiere comprobar, primero si las variables mencionadas influyen en la asignación del acento léxico en español. La forma en que lo hagan habrá de investigarse en trabajos posteriores, y elaborar así un modelo de acceso al léxico que incorpore el acento.

\section{Planteamiento de la investigación}

La población de los niños es ideal para estudiar cómo se usan los códigos fonológicos que intervienen en el proceso de acceso al léxico y, por lo tanto, el acento como un código fonológico más. En ellos el léxico ortográfico está poco desarrollado (Jiménez, Guzmán y Artiles, 1997) de modo que la ruta fonológica tiene más peso. Ello es cierto sobretodo en los idiomas transparentes como el español (Defior, Martos y Cary, 2002).

Según las pistas que indican el acento en español pueden realizarse las siguientes predicciones. En primer lugar, las palabras que lleven tilde tendrán que reconocerse antes. Ello es así porque en ellas no hay que aplicar ninguna regla, ya que el acento viene indicado de forma directa en la ortografía de la palabra. Del mismo modo, las llanas deberán tener ventaja, ya 
que su acento es el que se les presupone por defecto. Finalmente, las regulares (cuyo acento coincide con el que indica su estructura silábica) deberán leerse mejor que irregulares. De estas tres predicciones, la primera es la más difícil de comprobar. La tilde no sólo indica el acento, sino que también es un carácter gráfico. Por lo tanto, cualquier diferencia que se encuentre entre las palabras escritas con tilde y sin ella podría tener un origen tanto fonológico como ortográfico. Por ello, en este trabajo se estudiaron las dos últimas predicciones, escribiendo las palabras en mayúsculas y sin tilde para que de esa forma no hubiese ninguna pista explícita acerca de cuál debía ser su acento.

Según el modelo de Black y Byng (1986), de las dos predicciones que se ponen a prueba en esta investigación sólo debería cumplirse la que se refiere al acento más frecuente, de modo que se esperaría que las palabras llanas se reconociesen antes. Ello sería así porque, según este modelo, la búsqueda léxica comenzaría por este tipo de palabras. Sin embargo, la predicción que se haría según una versión del modelo DRC en la que se considerase la estructura silábica no sería tan simple. Tal y como ya se ha expuesto, la adaptación de dicho modelo al español conllevaría que se tuviera en cuenta la regla de que cualquier palabra sería llana salvo que su última sílaba sea pesada, en cuyo caso sería aguda. Por lo tanto, no cabría esperar que hubiera diferencias entre las palabras llanas y las agudas regulares, dado que éstas últimas terminarían en una sílaba pesada. Sin embargo, sí que deberían encontrarse entre las palabras regulares y las irregulares (p. e., casa y cajón, frente a sofá y túnel respectivamente).

Estas dos predicciones ya han sido comprobadas por Gutiérrez (2004), utilizando para ello una tarea de decisión léxica. Los resultados que obtuvo indicaron que las palabras regulares se reconocieron con menos errores. Probablemente, ello fue debido a que la estructura silábica hizo que dichas palabras se leyesen con un acento que no les correspondió (esto es, sofá y túnel leídas como SOfa y tuNEL), de modo que parecieran no-palabras. Para comprobarlo, en el experiento que se describe a continuación se utilizaron los mismos estímulos y se trabajó con una tarea diferente, en este caso de lectura de palabras en voz alta. Se prestó especial atención a las irregulares, en las que se esperaba que se produjesen errores de acento.

\section{Método}

Participantes: Colaboraron los mismos niños que tres meses antes habían tomado parte en la investigación de Gutiérrez (en prensa), esto es, 18 alumnos (12 niñas y 6 niños) de tercero de primaria del colegio Cristo Rey de Jaén. La edad media fue de 99.22 meses ( $\mathrm{SD}=3.6)$ y habían aprendido a leer siguiendo un método mixto, que combinaba características de los méto- 
dos global y fonético. Todos fueron evaluados como lectores normales por la profesora del centro.

Es posible que en un intervalo de tiempo tan largo la habilidad lectora de los niños haya mejorado, de forma que sea más difícil encontrar efectos fonológicos porque su léxico ortográfico se haya desarrollado. Sin embargo, dicho periodo coincidió con las vacaciones de verano, momento en el que no es habitual que se produzca ningún tipo de instrucción relacionada con la lectura. En todo caso, es más probable que, debido a la falta de práctica, se haya producido un deterioro. Así ocurre, por ejemplo, cuando se interrumpe por un tiempo la enseñanza de una segunda lengua, fenómeno conocido como attrition (Echeverría Munárriz, 1999).

Para evaluar el uso que los niños hicieron de los códigos fonológicos se utilizaron las escalas 5 (lectura de palabras) y 6 (lectura de palabras y no-palabras) de la batería PROLEC (Cuetos, Rodríguez y Ruano, 1996). Se contabilizaron los errores en la lectura de las nopalabra de ambas pruebas (véase el apéndice I). En el PROLEC no se dice qué es un error, así que se consideró como tal cualquier desviación de la lectura fluida de una palabra. Ello incluyó la lectura rectificando sobre la marcha (es-esbetulporión), el silabeo (es-be-tul-po-rión) y las pausas (esbetul-porión). De esta forma se tuvo una medida lo suficientemente sensible que permitió que se pudiera realizar un análisis de regresión posterior sobre los errores en la lectura de las no-palabras regulares e irregulares del experimento. A través de este análisis se obtendría un índice del origen fonológico de los errores. Es de esperar que haya una correlación positiva en el caso de las palabras regulares, cuya pronunciación se rige por las reglas fonológicas del español. Del mismo modo, es de esperar que en las irregulares la correlación no sea tan clara, o incluso sea negativa, dado que son las excepciones a las reglas.

Instrumentos: Se utilizó un ordenador 486 para presentar las palabras y una llave vocal para registrar las latencias de denominación vocal. Los experimentos se prepararon utilizando el programa MEL versión 2.01 (Micro Experimental Laboratory: Scheneider, 1989). Los participantes fueron evaluados a través de la batería PROLEC (Cuetos et al, 1996).

Materiales: Se seleccionaron 56 palabras bisílabas del diccionario de frecuencias de Justicia (1995), en un rango de 2 a 1445 ocurrencias (del total de las 255711 posibles). De este conjunto se escogieron 36 palabras regulares (en las que el acento coincidió con el que indicaba su estructura silábica). Éstas se asignaron a dos listas, 18 llanas y 18 agudas, igualadas en frecuencia léxica (Justicia, 1995) y frecuencia posicional de la primera sílaba (Justicia, Santiago, 
Palma, Huertas y Gutiérrez, 1996). Además, se elaboró una lista de 12 palabras regulares (de las que 4 también formaron parte del conjunto de las 36 anteriores) y otra de 12 irregulares, igualadas en las mismas variables y en su acento.

De las 56 palabras totales, 34 fueron llanas y 22 fueron agudas. Para lograr que hubiera el mismo número de palabras de ambos tipos se añadieron 12 palabras agudas de relleno. El apéndice I muestra las palabras utilizadas en este experimento.

Diseño: Hubo dos variables, acento (palabras llanas y agudas) y estructura silábica (palabras regulares e irregulares), que se manipularon intrasujeto y no se cruzaron factorialmente ${ }^{3}$.

Procedimiento: El experimento se hizo en dos sesiones realizadas en dos días. Cada una duró 20 minutos aproximadamente. En la primera, se pasó una prueba individual de competencia lectora, el PROLEC, para evaluar el uso que los niños hicieron de los códigos fonológicos durante la lectura de palabras. Concretamente, se pasaron las escalas 5 y 6 . Se midieron los errores en la lectura de no-palabras, de modo que más errores sugieren un menor conocimiento de las reglas fonológicas.

En la segunda sesión se utilizó una tarea estándar de lectura de palabras en voz alta. En cada ensayo, primero se escuchaba un pitido $(1000 \mathrm{~Hz})$ durante $50 \mathrm{~ms}$. Después se presentaba una señal $(+)$ que permanecía durante $450 \mathrm{~ms}$ en el centro de la pantalla del ordenador. La pantalla se borraba y quedaba en blanco durante otros $500 \mathrm{~ms}$. Finalmente, se presentaba la palabra que había que leer, que permanecía hasta que se la nombraba o hasta que transcurría un máximo de 3 segundos. Las palabras se presentaron en mayúsculas y sin tilde ${ }^{4}$, con un orden diferente para cada participante.

\section{Resultados}

En el ANOVA de los tiempos de reacción se excluyeron los ensayos en los que hubo algún error y aquellos no comprendidos en un intervalo de 200 a 2000 ms. En el caso de la variable acento, las diferencias fueron marginalmente significativas sólo por sujetos [F1(1, $17)=3.28, \mathrm{p}<0.10 ; \mathrm{F} 2(1,34)=2.43, \mathrm{p}>0.10]$. En el caso de los errores no hubo diferencias $[\mathrm{F} 1(1,17)<1 ; \mathrm{F} 2(1,34)<1]$. 


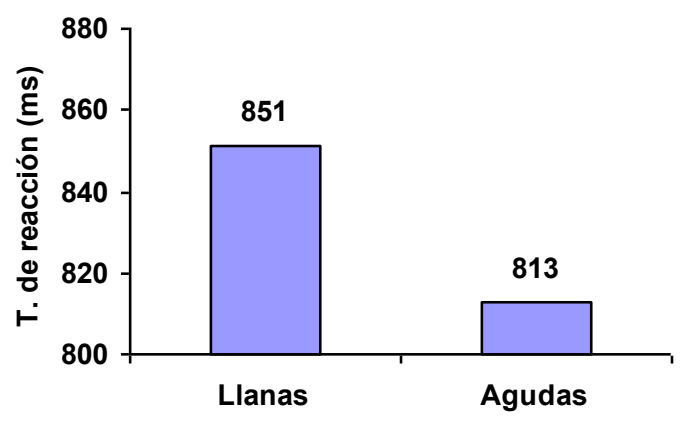

Gráfica 1. Medias de los tiempos de reacción por sujetos.

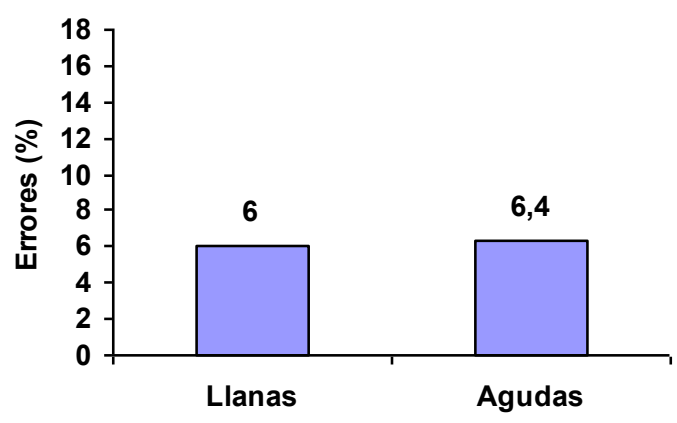

Gráfica 2. Medias de los tantos por ciento de errores por sujetos.

En el caso del ANOVA para los tiempos de reacción de la variable estructura silábica se encontró que no hubo diferencias significativas $[\mathrm{F} 1(1,17)<1 ; \mathrm{F} 2(1,34)<1]$, mientras que sí las hubo, aunque sólo por sujetos, en el caso de los errores $[\mathrm{F} 1(1,17)=22.13, \mathrm{p}<0.05$; $\mathrm{F} 2(1,34)=2.55, \mathrm{p}>0.10]$.

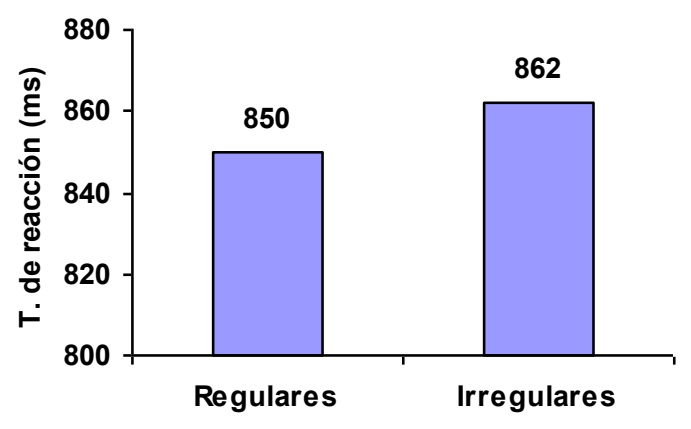

Gráfica 3. Medias de los tiempos de reacción por sujetos.

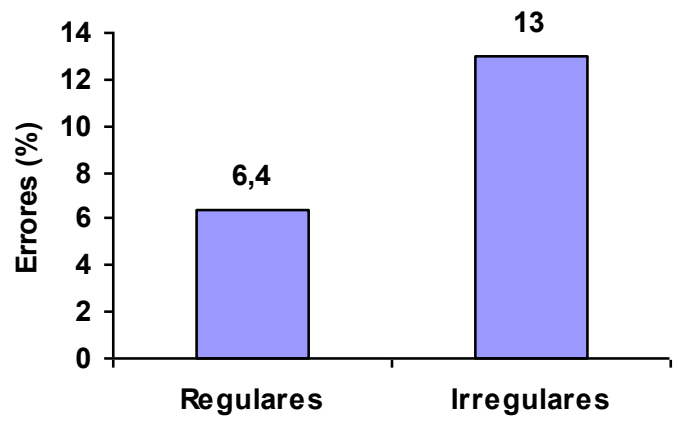

Gráfica 4. Medias de los tantos por ciento de errores por sujetos.

Con los errores se realizó un análisis adicional, de tipo descriptivo, para comprobar si se producían errores de acento (p. e., chándal leído como chándal). En las palabras regulares no hubo ningún caso de errores de este tipo, mientras que sí los hubo en 6 de las 12 palabras irregulares. Concretamente, en cárcel el $40 \%$ de los errores que se produjeron fueron de acento, 
en póster el porcentaje subió al 43\%, en túnel y chándal al 50\%, mientras que en sofá y café fue del 100\%.

Se realizó un análisis de regresión sobre los errores y a través de los sujetos para comprobar si hubo una relación entre el uso de códigos fonológicos y los errores cometidos en las palabras regulares e irregulares. La predicción de la que se partía era que a mayor fuese el uso de este tipo de códigos (es decir, a menos errores se cometan en la lectura de no-palabras) menor será la cantidad de errores que se produzcan en las palabras regulares (que siguen la reglas), mientras que en las irregulares no debería darse esta relación o incluso podría esperarse la contraria. Para estimar el uso de los códigos fonológicos se contabilizaron los errores cometidos en la lectura de las no-palabras de las pruebas 5 y 6 del PROLEC, de modo que a más errores se cometieron se consideró que el uso de dichos códigos fue menor.

Los resultados indicaron que la correlación fue positiva en las palabras regulares, $\beta=$ $0.59[\mathrm{~F}(1,16)=8.5, \mathrm{p}<0.05]$. El valor del coeficiente $\mathrm{R}^{2}$ ajustado fue de 0.30 , de modo que el porcentaje de varianza explicada alcanzó el 30\%. En las palabras irregulares la correlación también fue positiva, aunque sólo marginalmente significativa, $\beta=0.42[\mathrm{~F}(1,16)=3.37, \mathrm{p}<$ 0.10]. En este caso, el porcentaje de varianza explicada fue sólo del 12\%.

\section{Discusión}

En este experimento se ponían a prueba dos predicciones formuladas sobre la base de los factores que determinan la asignación del acento en español. Por una parte, se esperaba que las palabras llanas se leyesen más rápidamente y con menos errores que las agudas. Por otra parte, se esperaba que fueran las palabras regulares las que tuvieran ventaja frente a las irregulares. Según el modelo de Black y Byng (1986) debía cumplirse sólo la primera predicción, ya que la asignación del acento léxico se basa sólo en que, por defecto, se presupone que la mayoría de las palabras tienen el mismo acento. Sin embargo, si se tiene en cuenta la regla de que las palabras son llanas salvo que su última sílaba sea pesada, entonces las diferencias deberían encontrarse sólo entre las palabras que siguen esta regla y las que no la siguen.

Los resultados de este experimento no confirman la primera predicción, en incluso son contrarios a ella dado que las agudas fueron más rápidas. Aunque se trató de un efecto débil, marginalmente significativo sólo por sujetos, coincide con algunos resultados previos que se encuentran también en los adultos. En general, las latencias de denominación son mayores cuando el acento está al comienzo de la palabra (Gutiérrez, Palma y Santiago, 2001). Ello 
puede ser debido a una característica específica de la tarea de nombrar palabras, y es que en ella se puede planificar la producción de los primeros fonemas desde muy pronto, dado que en la ortografía de la palabra hay una buena cantidad de pistas que lo permiten. Así, por ejemplo, los grafemas presentes en la palabra pueden activar a las sílabas asociadas a dichos fonemas en el silabario (Levelt, Roelofs y Meyer, 1999). Dado que las sílabas átonas son las más frecuentes, es de esperar que se produzca un cierto retraso cuando la primera sílaba sea tónica, debido a los procesos competitivos que pudieran tener lugar.

En cuanto a la estructura silábica, los resultados son análogos a los que encontró Gutiérrez (en prensa). En las irregulares se cometieron más errores, dado que las reglas que relacionan el acento con la estructura silábica en este tipo de palabras llevaron a que se les asignara un acento equivocado. El análisis de regresión realizado apunta en la dirección de que estas diferencias se debieron a factores fonológicos. En el caso de las palabras regulares, hubo una correlación positiva con los errores en la lectura de las no-palabras. Por lo tanto, a menos errores de este tipo se produjeron, menos errores se cometieron en las palabras que siguieron las reglas fonológicas del español. Sin embargo, en el caso de las irregulares, la correlación, aunque positiva, sólo fue marginalmente significativa. Ello es lógico si se tiene en cuenta que este tipo de palabras no siguen todas las reglas, concretamente las que se refieren a la asignación del acento. La correlación que se encuentra en ellas pudo deberse a que, desde otro punto de vista, fueron regulares respecto a la aplicación de las reglas CGF.

En su conjunto, este patrón de resultados confirma la predicción de que la asignación del acento se hace teniendo en cuenta la estructura silábica. Por lo tanto sugiere que debería proponerse un modelo en el que se incorporase la estructura silábica como uno de los factores que determinan la asignación del acento en español. Además, se confirma la relevancia de la estructura silábica como una de las variables que influye en la lectura de los niños (Defior et al, 1996).

Finalmente, estos resultados sugieren que quizás debiera extenderse el concepto de conciencia fonológica para incluir variables tales como el acento y la estructura silábica. Podría hablarse entonces de un tipo de conciencia métrica que pudiera estar a la base del aprendizaje de la lectura y de sus dificultades. Dado que ambos factores son importantes para leer parecería lógico que hubiera que instruir a los niños acerca de su uso. Una de las tareas habituales de conciencia fonológica que podría combinarse con este tipo de entrenamiento es aquella que se refiere a la rima, que en español sería la rima silábica. Dado que la rima deter- 
mina el peso de la sílaba se podrían elaborar ejercicios en los que se manipulara el acento y la estructura de la rima final (p. e., $V$ para el acento llano, o $V C$ para el acento agudo). La tarea de los niños sería indicar si las palabras riman, considerando también su acento. La efectividad de este tipo de entrenamiento es una cuestión empírica que queda para ser comprobada en investigaciones futuras.

\section{Notas}

1. En español, el $90 \%$ de las palabras son llanas (Harris, 1995).

2. La sílaba es una unidad funcional para la lectura en español, tanto en adultos (Carreiras, Álvarez, y de Vega, 1993) como en niños (Jiménez, Guzmán y Artiles, 1997).

3. No hubo suficiente cantidad de palabras irregulares llanas y agudas para que se pudieran combinar las dos variables en un diseño factorial.

4. Aunque según las normas de la Real Academia (1991) las mayúsculas se rigen por las mismas reglas que las minúsculas, lo cierto es que es habitual ver escritas palabras mayúsculas sin tilde. Por ello, no esperábamos que hubiera diferencias debidas a este factor.

\section{Nota de los autores}

Este trabajo ha sido financiado parcialmente por la DGIGYT, a través del proyecto "Marcos estructurales y su utilización en percepción, producción y adquisición del lenguaje”, referencia BS02002-02993.

\section{Referencias}

Alonso-Cortés, A. (1998). El acento de los anglicismos y la estructura silábica del español. Boletín de la Real Academia Española, 78, 391-400.

Álvarez, C. J., De Vega, M., y Carreiras, M. (1998). La sílaba como unidad de activación léxica en la lectura de palabras trisílabas. Psicothema, 10, 371-386.

Álvarez, C. J., Carreiras, M., y De Vega, M. (2000). Syllable frequency effect in visual word recognition: evidence of sequential type processing. Psicologica, 21, 341-374.

Black, M., y Byng, S. (1986). Prosodic constraints on lexical access in reading. Cognitive Neuropsychology, 3, 369-409. 
Borowsky, R., Owen, W. J., y Fonos, N. (1999). Reading speech and hearing print: Constraining models of visual word recognition by exploring connections with speech perception. Canadian Journal of Experimental Psychology, 53, 294-305.

Cappa, S. F., Nespor, M., Ielasi, W., y Miozzo, A. (1997). The representation of stress: evidence from an aphasic patient. Cognition, 65, 1-13.

Carreiras, M., Álvarez, C., y De Vega, M. (1993). Syllable frequency and visual word recognition in Spanish. Journal of Memory and Language, 32, 766-780.

Colombo, L. (1992). Lexical stress effect and its interaction with frequency in word production. Journal of Experimental Psychology: Human Perception and Performance, 18, 987-1003.

Coltheart, M. (1978). Lexical access in simple reading tasks. Ed. G. Underwood (Ed.), Strategies of information processing (pp. 151-216). London: Academic Press.

Coltheart, M., y Rastle, K. (1994). Serial processing in reading aloud: Evidence for dual-route models of reading. Journal of Experimental Psychology: Human Perception and Performance, 20, 1197-1211.

Cuetos, F., Rodríguez, B., y Ruano, E. (1996). PROLEC: batería de evaluación de los procesos lectores de los niños de educación primaria. Madrid: TEA ediciones, S.A.

Cutler, A., y Clifton, J. R. (1984). The use of prosodic information in word recognition. En H. Bouma y D. G. Bouwhuis (Eds.), Attention and performance X. Control of language processes (pp. 183-196). London: Lawrence Erlbaum Associates Ltd.

Cutler, A., Dahan, D., y Donselaar, W. (1997). Prosody in the comprehension of spoken language: A literature review. Language and Speech, 40, 141-201.

Defior, S., Justicia, F., y Martos, F. J. (1996). The influence of lexical and sublexical variables in normal and poor Spanish readers. Reading \& Writing, 8, 487-497.

Defior, S., Martos, F., y Cary, L. (2002). Diferences in reading acquisition development in two shallow orthographies: Portuguese and Spanish. Applied Psycholingüistics, 23, 135-148.

Domínguez, A., y Cuetos, F. (1998). Similitud ortográfica y prosódica en el reconocimiento de palabras. II Congreso de la Sociedad Española de Psicología Experimental (SEPEX), Granada, 17-18 de diciembre.

Domínguez, A., y Cuetos, F. (2002). Lexical stress in visual word recognition: contrastive value in Spanish. Manuscrito en revisión (Journal of Memory and Language).

Domínguez, A., De Vega, M., y Cuetos, F. (1997). Lexical inhibition from syllabic units in visual word recognition. Language and Cognitive Processes, 12, 401-422.

Echeverría Munárriz, M. C. (1999). La regresión del aprendizaje del inglés en educación secundaria. Tesis Doctoral: Universidad de Granada.

Frost, R. (1998). Toward a strong phonological theory of visual word recognition: true issues and false trails. Psychological Bulletin, 123, 71-99. 
Gutiérrez, N. (2003). El acento léxico y su función en el reconocimiento de palabras escritas en adultos y en niños. Tesis doctoral no publicada. Universidad de Granada.

Gutiérrez, N. (en prensa). El acento y su función en el acceso al léxico de las palabras escritas: un estudio con niños. Manuscrito en revisión (Psicológica).

Gutiérrez, N., Palma, A., y Santiago, J. (1998). On the role of lexical stress in visual word recognition. Póster presentado en el XI congress of the European Society for Cognitive Psychology, en Jerusalem, Israel, 13-17 de septiembre.

Gutiérrez, N., Palma, A., y Santiago, J. (2001). La asignación del acento léxico en producción del lenguaje: diferencias entre palabras y dibujos. Póster presentado en el $V$ Simposio de Psicolingüística, Granada, 25-28 de Abril.

Haist, F., Song, A. W., Wild, K., Faber, T. L., Popp, C. A., y Morris, R. D. (2001). Linking sight and sound: fMRI evidence of primary auditory cortex activation during visual word recognition. Brain and Language, 76, 340-350.

Harris, J. W. (1995). Projection and Edge Marking in the computation of stress in Spanish. En J.A. Coldsmith (Ed.), The handbook of phonology theory (pp. 867-887). Cambridge, MA: Blackwell.

Hochberg, J. (1987a). Acquisition data and phonological theory: the case of Spanish stress. En J. Aske., N. Beery., L. Michaelis., y H. Filip (Eds.), Proceedings of the Thirteenth Annual Meeting of Berkeley Linguistics Society.

Hochberg, J. (1987b). The acquisition of word stress rules in Spanish. Papers and Reports in Child Language Development, 26, 56-63.

Jiménez, J. E., Guzmán R., y Artiles, C. (1997). Efectos de la frecuencia silábica posicional en el aprendizaje de la lectura. Cognitiva, 9, 3-27.

Justicia, F. (1995). El desarrollo del vocabulario. Diccionario de frecuencias. Granada: Servicio de Publicaciones de la Universidad de Granada.

Justicia, F., Palma, A., Santiago, J., Huertas, D., y Gutiérrez, N. (1996). La frecuencia silábica del español escrito por niños: estudio estadístico. Cognitiva, 8, 131-168.

Kelly, M. H., Morris, J., y Verrekia, L. (1998). Orthographic cues to lexical stress: effects on naming and lexical decision. Memory \& Cognition, 26, 822-832.

Levelt, W. J. M., Roelofs, A., y Meyer, A. (1999). A theory of lexical access in speech production. Behavioral and Brain Sciences, 22, 1-75.

Miceli, G., y Caramazza, A. (1993). The assignment of word stress in oral reading: evidence from a case of acquired dyslexia. Cognitive Neuropsychology, 10, 273-296.

Rastle, K., y Coltheart, M. (1998). Whammy and double whammy: The effect of length on nonword reading. Psychonomic Bulletin and Review, 5, 277-282.

Rastle, K., y Coltheart, M. (1999). Serial and strategic effects in reading aloud. Journal of Experimental Psychology: Human Perception and Performance, 25, 482-503.

Rastle, K., y Coltheart, M. (2000). Lexical and nonlexical print-to-sound translation of disyllabic words and nonwords. Journal of Memory and Language, 42, 342-364. 
Real Academia Española (1991). Esbozo de una nueva gramática de la lengua española. Madrid: Espasa-Calpe, S.A.

Sbisa, S., Zorzi, M., y Tabossi, P. (1998). Lexical stress effects in reading Croatian words. Póster presentado en el XI congress of the European Society for Cognitive Psychology, en Jerusalem, Israel, 13-17 de septiembre

Schneider, W. (1989). Micro Experimental Laboratory (MEL): An integrated software system for computerized experimentation for research and instruction on IBM PC-compatible computers. Behavior Research Methods, Instruments, \& Computers, 21, 240-244.

Soto-Faraco, S., Sebastián-Gallés, N., y Cutler, A. (2001). Segmental and suprasegmental mismatch in lexical access. Journal of Memory and Language, 45, 412-432.

Apéndice I

\begin{tabular}{|c|c|c|c|}
\hline \multicolumn{4}{|c|}{ Palabras } \\
\hline Llanas & Agudas & Regulares & Irregulares \\
\hline CABLE & COLLAR & COMPRA & CAFE \\
\hline LABIOS & CARTEL & LLUVIA & SOFA \\
\hline FERIA & CLAVEL & PIPAS & FUTBOL \\
\hline POLLO & PODER & TORRE & TUNEL \\
\hline CHICLE & PASTEL & RAIZ & ANGEL \\
\hline COMPRA & PORTAL & PAIS & CESPED \\
\hline LLUVIA & PERDIZ & BIBLIA & CARCEL \\
\hline PIPAS & SEÑOR & FOLIO & POSTER \\
\hline TORRE & TAMBOR & CUEVA & CHANDAL \\
\hline MEDIAS & BUZON & LENGUA & ARBOL \\
\hline UÑAS & TAPON & GAFAS & LAPIZ \\
\hline CARNE & BALON & SILLA & ALBUM \\
\hline PATIO & CAJON & \multicolumn{2}{|c|}{ Frecuencia léxica } \\
\hline HUEVOS & BOTON & $195.16(423.65)$ & $196.75(401.18)$ \\
\hline TABLA & JAMON & \multicolumn{2}{|c|}{$\begin{array}{l}\text { Frecuencia de la primera sílaba } \\
\end{array}$} \\
\hline LETRA & MELON & $63.83(67.95)$ & $73.66(99.70)$ \\
\hline JARRA & CORDON & \multicolumn{2}{|c|}{ Frecuencia de la sílaba final } \\
\hline GORRA & CARTON & $17.66(33.09)$ & $5.91(6.08)$ \\
\hline \multicolumn{2}{|c|}{ Frecuencia léxica } & & \\
\hline $45.39(31.63)$ & $44.55(32.07)$ & & \\
\hline \multicolumn{2}{|c|}{ Frecuencia de la primera sílaba } & & \\
\hline $84.55(90.50)$ & $92(99.74)$ & & \\
\hline \multicolumn{2}{|c|}{ Frecuencia de la sílaba final } & & \\
\hline $20.50(24.98)$ & $8.66(6.27)$ & & \\
\hline
\end{tabular}

Los valores numéricos son medias y, entre paréntesis, se muestra la desviación típica. El valor de la frecuencia silábica representa las palabras diferentes que compartieron la sílaba en esa posición (se trata, por lo tanto, de un dato tipo type). Se prefiere ese dato a los valores tipo token porque está en una escala que facilita la comparación entre condiciones.

\section{Lectura de las no-palabras del PROLEC}

\begin{tabular}{|r|r} 
Prueba 5 & Prueba 6 \\
$4.27(2.67)$ & $5.66(1.91)$ \\
\hline
\end{tabular}

El primer valor corresponde a la media de los errores cometidos. Entre paréntesis se muestra la desviación típica. 